\title{
The I in COVID: The importance of community and patient involvement in COVID-19 research
}

\author{
Author: Anenta Ratneswaren ${ }^{\mathrm{A}}$
}

The call for community and patient involvement in the COVID-19 response is yet to be heard and answered. There are practical and ethical reasons for researchers not to neglect patient and public involvement (PPI), which has become an important cornerstone of UK-based clinical research. There has been a commendable effort towards driving evidence-based research, particularly through clinical trials in the UK. This article presents a brief background to PPI and points for consideration for clinical researchers currently active in or planning COVID-19 research.

KEYWORDS: Patient and public involvement, community, engagement, clinical trials, COVID-19

DOI: $10.7861 /$ clinmed.2020-0173

\section{Introduction}

The need for community and patient involvement in research has never been greater. COVID-19 represents the need and the opportunity to make sure that all clinical research has meaningful patient and public involvement (PPI), whether it be at the design, undertaking, dissemination or evaluation stages of the research cycle.

COVID-19 has humbled clinicians across the world, has challenged dogma and has left us with many unanswered questions. The cumulative unknown effects of health service choices, social behaviours and pathological processes necessitate a consultative approach to health policy and research that is inclusive of public voices.

There are uncanny parallels between COVID-19 and the HIV pandemic over the last 40 years. A valuable, and perhaps intuitive, lesson from the HIV experience that has frequently been cited over recent weeks is the need for community engagement for an effective COVID-19 response. ${ }^{1}$

\section{Historical background}

It is worth remembering that the origins of PPI can be traced back to voices such as educator Paolo Friere, and his concept of citizen 'conscientisation', ${ }^{2}$ and philosopher Michel Foucault, and

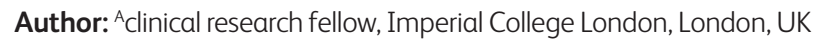

his discourse on power and knowledge as social constructs and on the doctor-patient relationship. ${ }^{3}$ Thus, the use of PPI should be seen as a transformative process whereby the public can be empowered, whether through the redistribution of power or the development of social capacity.

The UK is a pioneer in demonstrating a national commitment to public involvement. 'No decision about me, without me' underpinned the vision of a public and patient-centred NHS that was set out by the UK Government's 2010 white paper, Equity and excellence: liberating the NHS. ${ }^{4}$ The 'patient revolution' has filtered through to all aspects of healthcare design and delivery, and regulations stipulate that PPI must be part of the design, development and applied aspects of all NHS organisation work. PPI has evolved over several decades now and the role of PPI in clinical research is reinforced through the National Institutes of Healthcare Research (NIHR)'s dedicated organisation, INVOLVE.

Funding bodies, including charities, have long mandated evidence of PPI in grant applications, and they should not relax this for COVID-19 research. Patient review to complement traditional peer review has recently been introduced by a number of academic journals, many of which also request authors provide a tweet written for a public audience, as well as for the academic community, to encourage public engagement with healthcare science.

While they may overlap conceptually and practically, there is an important distinction to be made between involvement and engagement. Involvement refers to the active involvement of people in carrying out research projects, whereas engagement refers to the broader information and knowledge about research that is shared with the public. Participation in research, meanwhile, describes people providing data for analysis, ie research study participants.

\section{The importance of inclusiveness}

Evidence has emerged that in the UK the ability to adopt and comply with certain interventions against COVID-19, such as the ability to work from home and to self-isolate, is a privilege. ${ }^{5}$ Atchison and co-authors found that those with the lowest household incomes were less likely to be able to work from home and three times less likely to be able to self-isolate. ${ }^{5}$ Arguably, this is a reflection of the often-systematic tendency of policy and research to overlook the most vulnerable in society. These findings and the current concern over the higher risk of death in black, Asian and minority ethnic (BAME) groups highlight the importance of capturing socioeconomic status, ethnicity and occupation in COVID-19 clinical research. 
PPI can be used to hear the voices of the marginalised and the vulnerable in clinical research. However, PPI research has previously been criticised for not sufficiently representing those with lower health status and poorer access to services. ${ }^{6}$ There are several practical ways to mitigate this; for example, being mindful of this potential bias, working with grass-roots community-based organisations and leaders, and developing written information and scripts to ensure that language is clear for a lay audience.

On a systematic level, PPI is criticised as an exercise that is too subjective and lacking in legitimate representativeness. It may be worth including those with and without lived experience of the condition, including family and carers. In clinical research to date, under-represented groups in PPI reflect under-represented groups in clinical trials. This includes adolescents and young adults and those from ethnic minorities and less-advantaged socio-economic groups. This may reflect the traditional ways of inviting participation through established patient networks. Social media and community groups can be used to target under-represented groups.

It is important to remember that PPI contributors may have specific training needs for particular involvement activities, for example to develop skills in understanding terminology, reviewing documents, managing emotions or contributing effectively in meetings. Choices around inclusion and exclusion must be balanced with the choice of involvement methodology, for example ethnographic study, interviews, or surveys, as well as time and resource constraints.

\section{Impact}

The National Standards for Public Involvement are six standards for PPI to be benchmarked against.? The fifth standard directly calls for meaningful evaluation of the impact of PPI. This includes cost-effectiveness, as significant time and financial resources (up to $10 \%$ of a clinical trial's cost) may be required. The limitations to date for measuring the impact of PPI work have been the emphasis on the intrinsic value of PPI, the need for a common understanding of PPI in practice, and methods to capture its full value.

Any COVID-19 PPI work should therefore include an evaluation strategy. There is no consensus on how this is best done, and indeed whether it should be qualitative or quantitative in nature. An impact log is a simple approach to recording PPI outcomes, but a more structured method of reflecting on and recording outcomes is the 'cube' framework of four domains. ${ }^{8} \mathrm{~A}$ useful and validated approach is the Public Involvement Impact Assessment Framework (PiiAF), which is a two-part tool for exploring impact and development of an assessment plan, which was itself constructed with public involvement ${ }^{8}$ and is recommended by the NIHR.

Principal investigator leadership is a key influence on impact that is under-recognised, ${ }^{9}$ and this has a practical implication, especially for rapid research. Building collaborative relationships between individual researchers and members of the public takes time, but there are several grassroots organisations that can be utilised to facilitate and maximise impact at all stages of the research cycle. ${ }^{10}$ For example, NIHR invoDIRECT is an online resource of networks, groups and organisations for researchers, and VOICE is a large network of citizens that connects members and researchers.

\section{Clinical trial research}

PPI interventions have been shown to improve recruitment and retention in clinical trials. ${ }^{11}$ Without emphasising the role of clinical

\section{Box 1. Useful websites}

$>$ NIHR INVOLVE: invo.org.uk

$>$ NIHR invoDIRECT: invo.org.uk/communities/invodirect

$>$ VOICE: voice-global.org

$>$ NIHR COVID-19 studies: nihr.ac.uk/covid-studies

$>$ European Patients Academy: eupati.eu

trials over other approaches in the effort to mitigate the health and social costs of COVID-19, clinical trials are used here as an illustration of how PPI can be incorporated even for urgent work. On 3 April 2020, Chris Whitty, chief medical officer for England, openly called for the recruitment of patients into nationally prioritised clinical trials on COVID-19.12 The Medicine and Healthcare products Regulatory Agency (MHRA) has developed procedures for rapid scientific advice, reviews and approvals, and was able to authorise clinical trial applications within a week during the Ebola outbreaks. ${ }^{13}$ There are 24 registered COVID-19 trials active in the UK to date (see www.clinicaltrialsregister.eu and www.clinicaltrials.gov), with 21 recognised as nationally prioritised studies.

For example, C-19ACS is a registered trial recruiting at Imperial College London NHS Trust. Based on direct anecdotal evidence from carer involvement, a survey was conducted to explore the views of COVID-19 research in delirium patients, which is increasingly being reported as a strong association by clinicians across the world.

Within 12 hours it was possible to design and deliver a survey of 120 responses, which was considered an adequate sample size for the purpose. In this time, it was possible to undergo a round of clinical peer review, a round of public piloting, implement feedback, invite participants and report results using Google Forms, a free online survey tool. Though Google Forms is itself GDPR-compliant and tools are available to store and send encrypted data, it must be used in a GDPR-responsible way. This is an example of consultation-type PPI and may be considered a low-level form of involvement. Nevertheless, it demonstrates that PPI work can rapidly incorporated during the COVID-19 research response. Significantly, a third of survey respondents were over the age of 65 years old and the use of an online survey requiring an email address was not seen as a barrier. Moreover, nearly half of the participants were willing to be contacted again. In another survey, Pristera and co-authors found that over two-thirds (68.6\%) of people wanted to or might want to be more involved with those working on the UK's response to the outbreak. ${ }^{14}$

The pharmaceutical industry in the UK has a growing role in PPI activities, especially in clinical trial research. The pharmaceutical industry is an important stakeholder and potential partner in the global response to COVID-19. In planning a PPI activity with industry, it is important to have a shared understanding of the goals and partnership. The Innovative Medicines Initiative (IMI) European Patients Academy (EUPATI) is a pan-European consortium focused on educating and training the public to ensure they can contribute to medicine development and offers a useful toolkit for approaching PPI work with a commercial sponsors.

\section{Conclusions}

With the level of media exposure and impact of COVID-19 on the public's everyday life, the public are 'pumped and primed' to be involved in research into the disease. Use of technology and 
creative ways of working can only serve to accelerate patient and public involvement in research. Though it may be new territory for some, requiring new methods, we have a duty to recognise the importance of PPI in our work, and there is guidance ${ }^{7.8}$ and other free and online resources available, including practical toolkits and online communities (see Box 1 for useful websites). We should not waste this opportunity to engage and involve the public in shaping the future of their health and their healthcare.

\section{References}

1 UNAIDS. Rights in the time of COVID-19: Lessons from HIV from an effective, community-led response. UNAIDS, 2020. Available from www.unaids.org/en/resources/documents/2020/humanrights-and-covid- 19.

2 Freire P. Cultural action and conscientization. Harvard Educational Review 1970;40:452-77.

3 Foucault M. The birth of the clinic: an archaeology of medical perception. Tavistock Publications, 1973

4 Department of Health. White Paper: Equity and excellence: liberating the NHS. DOH, 2010. Available from https://assets.publishing. service.gov.uk/government/uploads/system/uploads/attachment_ data/file/213823/dh_117794.pdf [Accessed 11 April 2020].

5 Atchison C, Bowman L, Vrinten C et al. Perceptions and behavioural responses of the general public during the COVID-19 pandemic: $\mathrm{a}$ cross-sectional survey of UK adults. MedRxiv 2020.04.01.20050039 (doi: 10.1101/2020.04.01.20050039).

6 Ocloo J, Matthews R. From tokenism to empowerment: progressing patient and public involvement in healthcare improvement. BMJ Qual Saf 2016;25:626-32.

7 National Standards for Public Involvement. NIHR, 2019. Available from www.invo.org.uk/posttypepublication/national-standards-forpublic-involvement/
8 Kok M. Guidance document: evaluating public involvement in research. UWE Bristol, 2018. Available from www.phwe.org.uk/ wp-content/uploads/Guidance-on-evaluating-Public-Involvementin-research.pdf [Accessed 11 April 2020].

9 Evans D, Coad J, Cottrell $\mathrm{K}$ et al. Public involvement in research: assessing impact through a realist evaluation. Health Services and Delivery Research 2014;2:36.

10 Hoddinott P, Pollock A, O'Cathain A et al. How to incorporate patient and public perspectives into the design and conduct of research. F1000Research 2018:7:752.

11 Crocker J, Ricci-Cabello I, Adwoa Parker A et al. Impact of patient and public involvement on enrolment and retention in clinical trials: systematic review and meta-analysis. BMJ 2018;363:k4738.

12 Atherton F, Calderwood C, McBridge M, Whitty C, Powis S. Novel coronavirus: clinical trials [letter to NHS trusts]. Available from www.nihr.ac.uk/documents/news/the-importance-of-COVID-19clinical-trials.pdf [Accessed 11 April 2020].

13 Medicines and Healthcare products Regulatory Agency. Guidance: Clinical trials applications for Coronavirus (COVID-19). Available from www.gov.uk/guidance/clinical-trials-applications-for-coronaviruscovid-19 [Accessed 11 April 2020].

14 Pristera P, Papgeorgiou V, Kaur M et al. Report 14: Online community involvement in COVID-19 research and outbreak response: early insights from a UK perspective. Available from www.imperial. ac.uk/media/imperial-college/medicine/mrc-gida/2020-04-03COVID19-Report-14.pdf [Accessed 11 April 2020].

Address for correspondence: Dr Anenta Ratneswaren, Block B, Hammersmith Hospital, Du Cane Road, London W12 OHS, UK. Email: anenta.ratneswaren@imperial.ac.uk 